\title{
WUNDREINIGUNG
}

\section{Leitungswasser genügt vollauf}

Auf der Suche nach preiswerten und effektiven Mitteln zur Wundreinigung sind Forscher der Cochrane Wounds Group nun beim Leitungswasser angelangt. In den einschlägigen Studien wurde der reinigende Effekt von Leitungswasser im Vergleich zu isotonischer Salzlösung an Risswunden, offenen Frakturen, chronischen und chirurgischen Wunden getestet. Primärer Endpunkt war die Häufigkeit von Wundinfektionen. Ergebnis: kein Unterschied - bis auf einen Fall, nämlich akute Wunden von Erwachsenen. Hier erwies sich das Leitungswasser sogar als signifikant überlegen, das Infektionsrisiko lag im Mittel um $17 \%$ niedriger als nach Spülungen mit Kochsalz. In drei Studien wurde übrigens untersucht, ob sich nicht gereinigte Wunden öfter infizieren als solche, die mit Leitungswasser gespült werden. Auch hier eine klare Antwort: Nein. Eine noch preiswertere Methode zu finden, dürfte selbst Cochrane-Forschern schwerfallen.

Ann Emerg Med 2012; doi: 10.1016/j.annemergmed.2012.06.011

\section{SCHWERE INFEKTIONEN BEI KINDERN}

\section{Auf das Bauchgefühl des Arztes ist Verlass}

In eine Beobachtungsstudie wurden 3869 Kinder und Jugendliche bis 16 Jahre eingeschlossen, die wegen einer akuten Erkrankung bei einem Kinderarzt oder Allgemeinmediziner vorgestellt wurden. Neben den klinischen Kriterien wurde für jedes Kind auch das „Bauchgefühl" des untersuchenden Arztes zur Schwere der Erkrankung festgehalten. Dabei wurde eine Infektion als schwer definiert, wenn innerhalb von mindestens 24 Stunden eine Klinikeinwei- sung nötig war. Von den 3369 Kindern, bei denen aufgrund ihres klinischen Erscheinungsbildes zunächst keine ernsthafte Erkrankung vermutet worden war, wurden sechs später dennoch mit einer schweren Infektion stationär aufgenommen. Hätten die Ärzte auf ihr Bauchgefühl gehört, hätte dies zwar in 44 von 3363 Fällen Fehlalarm bedeutet, aber zwei der sechs ernsthaft Erkrankten hätten davon profitiert. BMJ 2012, online 25. September; 345: e6144

\section{OVARIALKREBS}

\section{Diagnose mit Stift und Papier}

Papier, Bleistift und fünf Minuten Zeit - mehr ist nicht nötig, um sich als Hausarzt darüber zu informieren, ob eine Patientin womöglich an einem Ovarialmalignom leidet. Forscher des Fred Hutchinson Cancer Research Center in Seattle haben einen Fragebogen, "Symptom-Index" genannt, konzipiert, mit dem sich Risikokandidatinnen für ein Karzinom der Eierstöcke identifizieren lassen. Sie konzentrierten sich dabei auf einige wenige Symptome, die seit höchstens einem Jahr bestehen und öfter als zwölfmal im Monat auftreten: Geblähtsein, vergrößerter Bauch- umfang, Schmerzen im Becken oder Abdomen, Probleme beim Essen und Völlegefühl. Eine vorangegangene Studie hatte ergeben, dass 57\% der Frauen mit Frühstadien und $80 \%$ der Frauen mit fortgeschrittenen Stadien eines Ovarialkarzinoms solche Beschwerden haben. Für die Aussagefähigkeit des Tests sei es wichtig, so die Forscher, nach gegenwärtigen Symptomen zu fragen, nicht nach solchen, die irgendwann in der Vergangenheit aufgetreten sind.

Open Journal of Obstetrics and Gynecology 2012; 2:183-191. doi: 10.4236/ojog.2012.23037

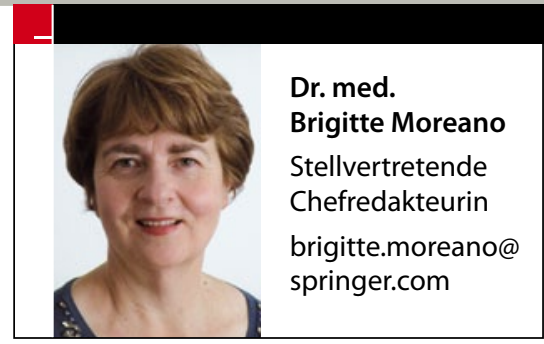

\section{LUNGENEMPHYSEM}

\section{Wegbereiter des Bronchialkarzinoms?}

Um dem Zusammenhang zwischen entzündlichen Lungenerkrankungen und Lungenkrebs auf den Grund zu gehen, analysierten Wissenschaftler des International Lung Cancer Consortiums die Daten von 17 Studien. Demnach ist bei Patienten mit einem Emphysem das Bronchial-Ca.-Risiko um das Zweieinhalbfache im Vergleich zu Lungengesunden erhöht. Bei Patienten mit chronischer Bronchitis beträgt der Faktor 1,47 und mit Tuberkulose 1,48. Bei einer Pneumonie in der Anamnese ist das Risiko für Lungenkrebs um den Faktor 1,57 erhöht.

Am J Epidemiol 2012, online 17. 9; doi: 10.1093/aje/ kws 151

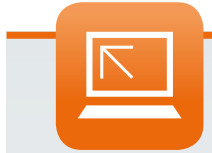

Themenvielfalt auf springermedizin.de

Unser Redaktionstipp

Das empfiehlt Ihnen die MMWRedaktion auf springermedizin.de:

- Alle Highlights vom Euro-

päischen Diabetes-Kongress

$($ EASD) $>3421230$

- Video-Interview zur Beschnei-

dungsdebatte 3541410 (Ein

Beitrag zu diesem Thema erwartet

Sie auch in der nächsten MMW!)

- Video-Interview: Brauchen

Männer einen Männerarzt? 3537776

(Geben Sie die ID-Nummer in die Suche ein.) 